\title{
Result Analysis of Noise Removal in ECG Signal using Wavelet Decomposition Technique
}

\author{
Anamika Rajput \\ M.Tech.Scholar \\ Technocrats Institute of \\ Technology- Advance \\ Bhopal (M.P.)
}

\author{
Pankaj Soni \\ Professor \\ Technocrats Institute of \\ Technology- Advance \\ Bhopal (M.P.)
}

\author{
Anshul Awasthi \\ Professor \\ Technocrats Institute of \\ Technology- Advance \\ Bhopal (M.P.)
}

\begin{abstract}
Now-a-days Cardio vascular diseases become huge threat to the lifetime of human beings. ECG is one in all the simplest technique to give clear information about cardiac arrhythmia. The electro-cardiogram may be a technique of recording bioelectric currents generated by the heart that is useful for diagnosing several cardiac diseases. The feature extraction and denoising of ECG are extremely useful in cardiology. ECG may be a non-stationary signal and it is used for the first diagnosis of cardiac abnormalities like arrhythmia, MI and conduction defects. However the ECG signals usually contaminated by different noises. The ECG signal should be denoised to remove all the noises like Additive White Gaussian noises.
\end{abstract}

In latest years, electro-cardiogram (ECG) acting a commanding role in heart illness diagnostics, Human pc Interface (HCI), stresses and emotional states valuation, etc. Generally, ECG signals exaggerated by noises like baseline wandering, power line interference, electromagnetic intervention, and high frequency noises throughout information acquirement.

\section{Keywords}

Electro-cardiogram (ECG), line interference (PLI), Symlet wavelet transform, noise, signal to noise ratio (SNR), thresholding, signals denoising.

\section{INTRODUCTION}

The ECG signals are examined wide for the diagnosis of the many cardiac diseases. The signals are traced using non invasive electrodes that are placed on the chest and limbs. The guts muscle cells that are located in atria and ventricles contract generating electrical pulses that are then traced by the ECG. The ECG signals of a standard heart beat contain 3 parts: $\mathrm{P}$ wave, QRS advanced and $\mathrm{T}$ wave. The $\mathrm{P}$ wave represents the atrial contractions. QRS complicated denote ventricle contractions. [1] The third wave in an ECG is that the $\mathrm{T}$ wave. This can be made once the ventricles are re polarizing. These waves show ample vary of deformities within the ECG signal.

The ECG recordings that are obtained by placing electrodes are usually contaminated by many sorts of noises. These include power line interference (PLI), base line wander, muscle contraction and motion artifacts. PLI represent the most a part of the distortions at 50-60 Hz. Motion artifacts are the transient baseline changes caused by mismatching of impedance between the electrodes and also the skin. [2] Baseline wander is that the continuous drifting of the ECG Signal from the baseline. it's mainly caused by respiration and increased body movements. [3] The distortions are created by the noise from the recorder, electronic devices and electrostatic potentials. [4] The interference at intervals the ECG signal affects the analysis and also the detection of QRS complex. Hence, for the proper diagnosis of the cardiac disease the ECG signal ought to be clean and noise free. The most requirements for noise removal of ECG Signal are: 1. ECG denoising methodology ought to preserve the ECG characteristic waves and it shouldn't disturb the sharp ECG peaks. 2. ECG denoising improves SNR. [5]

ECG noise removal involves multiple filters with numerous techniques. Digital filters are most popular due to its high stability, frequency deviation range; pc based style, low price implementation and increased reliability. Digital filters are usually classified into two: FIR and IIR filters. The low pass filter helps limiting the artifact for routine cardiac rhythm observation. It helps within the reduction of PLI. Because the viscus signals are low frequency signals, a FIR filter is that the right option to remove the noise.

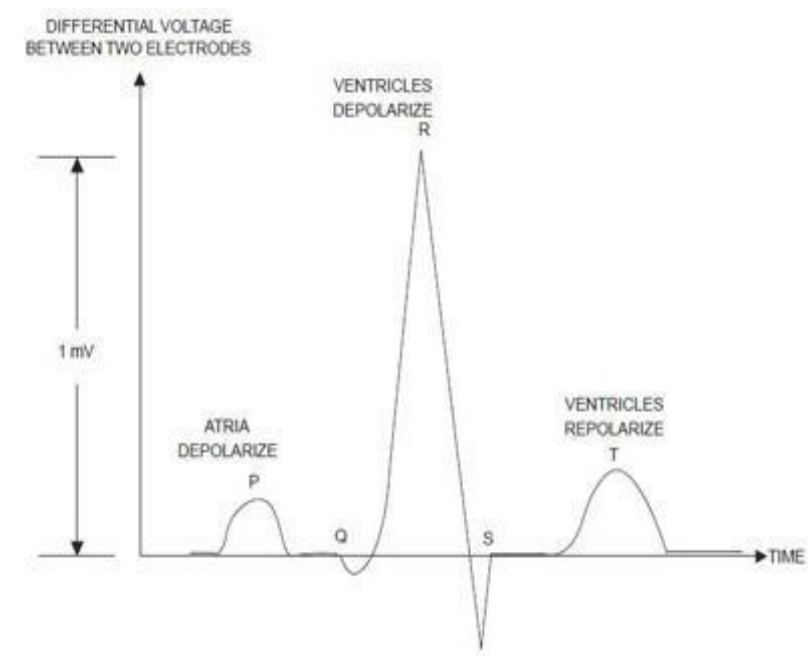

Fig.1 Typical ECG Signal

\section{THEORY}

\subsection{Noises in ECG}

While collection and transmission, the ECG gets corrupted because of differing types of artifacts and interferences. ECG noise will be classified as:

\subsubsection{Power line interference}

Power line interference (Fig.2 consists of 50-60 Hz $\pm 0.2 \mathrm{~Hz}$. This noise is usually modeled as sinusoids [15] a mix of sinusoids with a frequency of $50-60 \mathrm{~Hz}$ (fundamental) with 
harmonics and therefore the amplitude up to 500th of the complete peak-to-peak ECG amplitude.

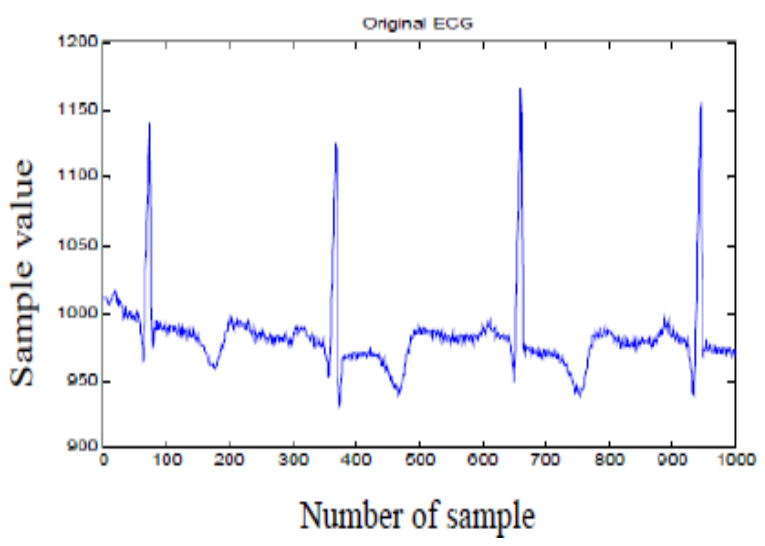

Fig. 2 the ECG Signal With Power Line Interference

\subsubsection{Electrode pop or contact noise}

This noise is caused by the loss of contact between the electrodes and also the skin, that effectively disconnects the measurement system and generates giant artifacts since the ECG signal is typically capacitive, coupled to the system. The characteristics of this noise signal include the amplitude of the initial transition, the amplitude of the sixty $\mathrm{Hz}$ part and also the constant time of the decay. Typical parameters are one second period, most recorded output amplitude, and a frequency of between $50-60 \mathrm{~Hz}$.

\subsubsection{Data collecting device noise}

Data collection device noise artifacts are generated by the signal process hardware, like signal saturation.

\subsubsection{Electrosurgical noise}

Electrosurgical noise is generated by different medical equipment present within the patient care environment at frequencies between $100 \mathrm{kHz}$ and $1 \mathrm{MHz}$, lasting for about 1 to 10 seconds.

\subsubsection{Channel noise}

Poor channel conditions can even introduce noise to the ECG once ECG is transmitted. It's sometimes modeled as white Gaussian noise (random and not systematically planned with mean zero), that contains all frequency elements.

\section{PROPOSED METHOD}

We introduce the energy efficient Power Spectral Analysis of ECG signal by utilizing DWT and FFT. Initially, we tend to found a site (i.e. Wavelet) within which the input signal is accurately sparse and that we continue by expressing it during this domain by utilizing an appropriate transformation (i.e. DWT). The few large signal parts are most significant or relevant for the whole signal quality, whereas different signal parts and also the corresponding computations will probably be skipped or pruned for major complexness reduction.

The noted signal is usually adulterated by noise and artifacts that may be under the frequency band of interest and manifest with same characteristic because the ECG signal itself. In arrangement to extract noisy ECG signals, we tend to need to execute the elemental electrocardiogram signal. ECG signal process will be roughly classified into 2 ways that:
1) Preprocessing

2) Feature extraction.

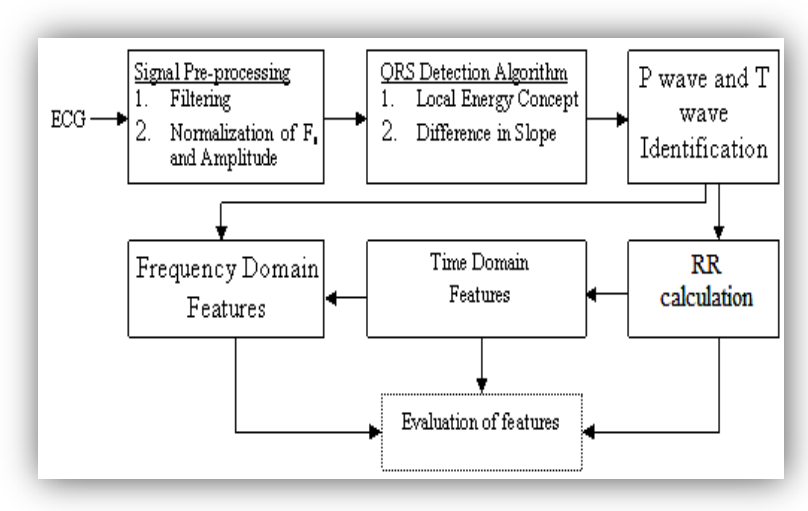

Fig.3 proposed system

\subsection{Pre-Processing}

The pre-processing of raw ECG signal is needed for removal of noises like muscle noise, $60 \mathrm{~Hz}$ interference, baseline wander and T-wave interference, etc. Pre-processing stage involves normalization and filtering. Within the normalization method the amplitude of the signal is normalized initial then it's passed through band pass filter. The operate of band pass filter is noise rejection. The desirable pass band to maximize the QRS energy is approximately $5-15 \mathrm{~Hz}$. The raw ECG signal, the signal when normalization and also the normalized signal, when passing through band pass filtering.

\subsection{Feature Extraction}

The variations in ECG options like QRS-complex morphology, ST-segment deviation and T-wave alternation is used for ischemic episode detection. initial feature towards the ischemic episode detection method is that the determination of QRS-complex. The R-peak that has the tallest peak in ECG signal is initial determined in QRS-complex. Then the lowest peak $\mathbf{S}$ is decided followed by Q-peak. The appropriate methodology used for finding QRS-complex is based on $\mathrm{J}$. Pan and J. Tompkins algorithmic rule [9]. Different options like P-wave location, J-point location, T-wave, TON and TOFF locations, iso electric line and ST-segment location were detected using the previously located Q-, R- and S-wave peaks.
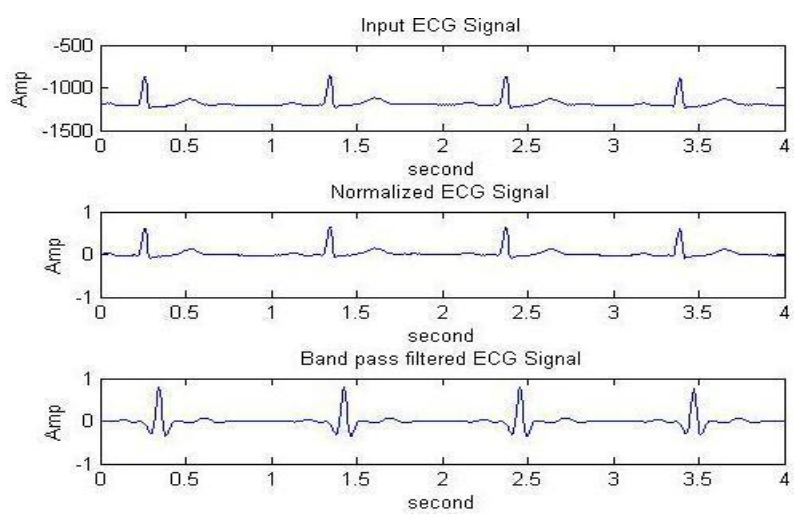

Fig.4 Normalized and filtered ECG signal 


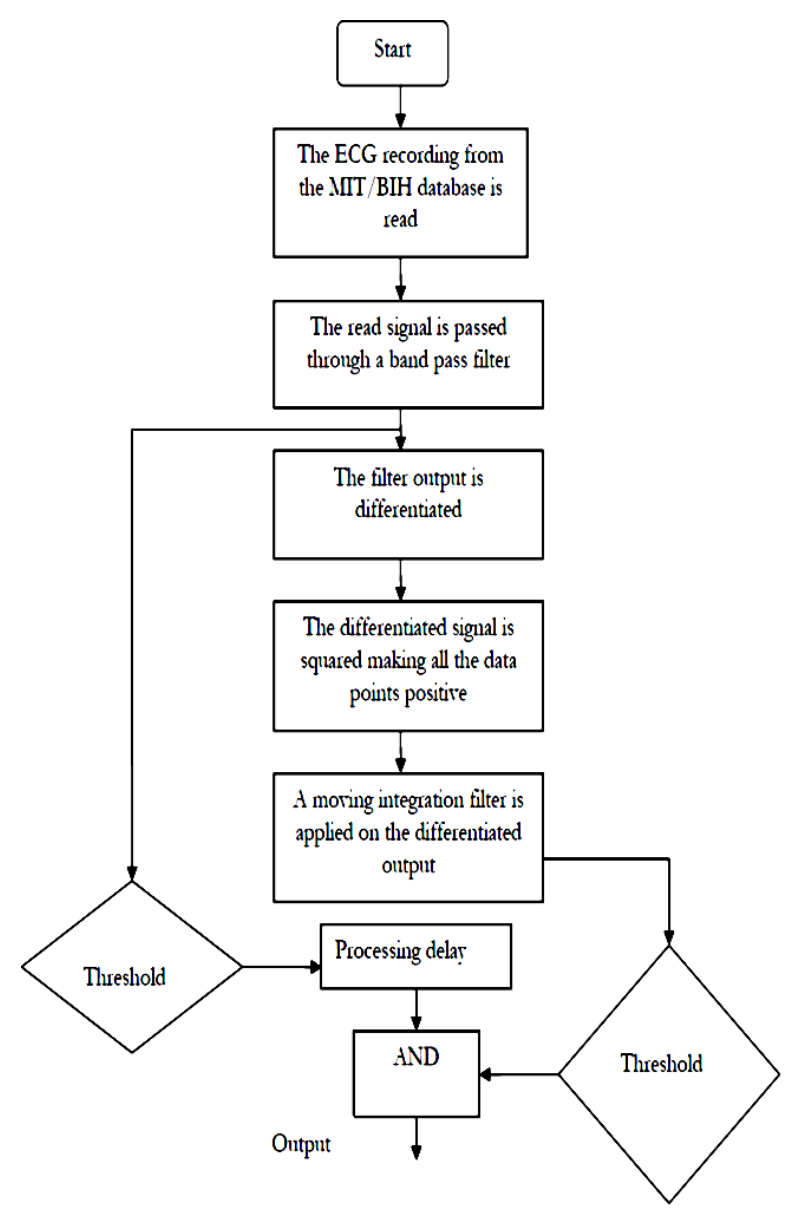

Fig.5 Flow diagram of proposed methodology

\section{RESULT}

This section focus on the outputs obtained at each step. The proposed algorithm is evaluated with the database consist of ECG signals.

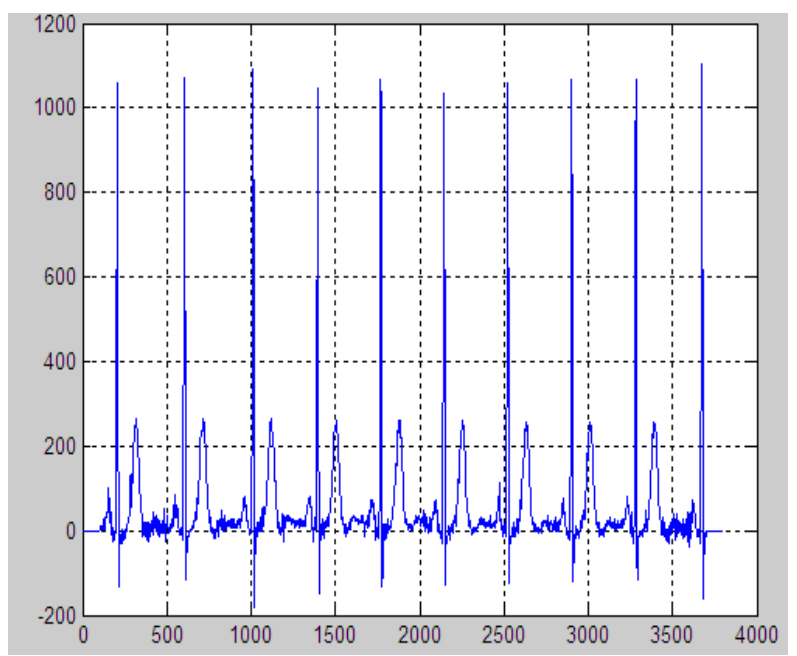

Fig.6 Actual signal

This fig.6 shows the actual ECG signal that is input signal

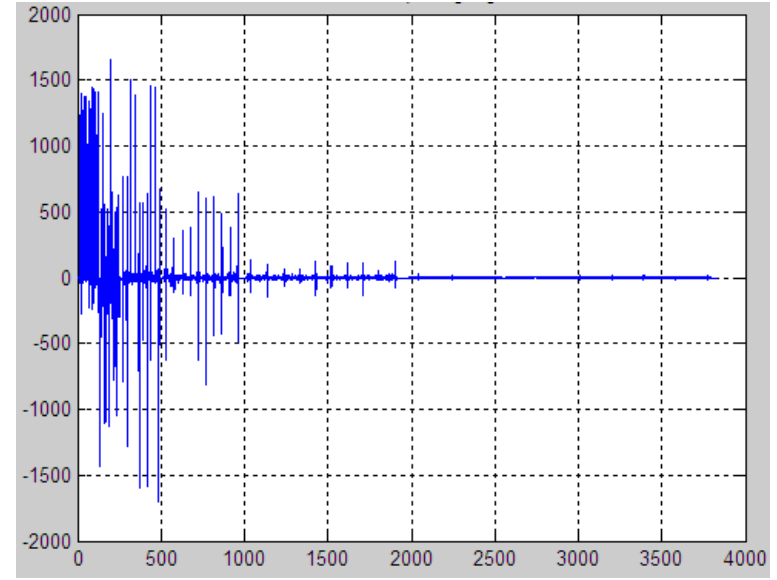

Fig.7 ECG decomposing signal

This fig. 7 shows the ECG decomposing signal. After process the decomposition method this signal is obtained.

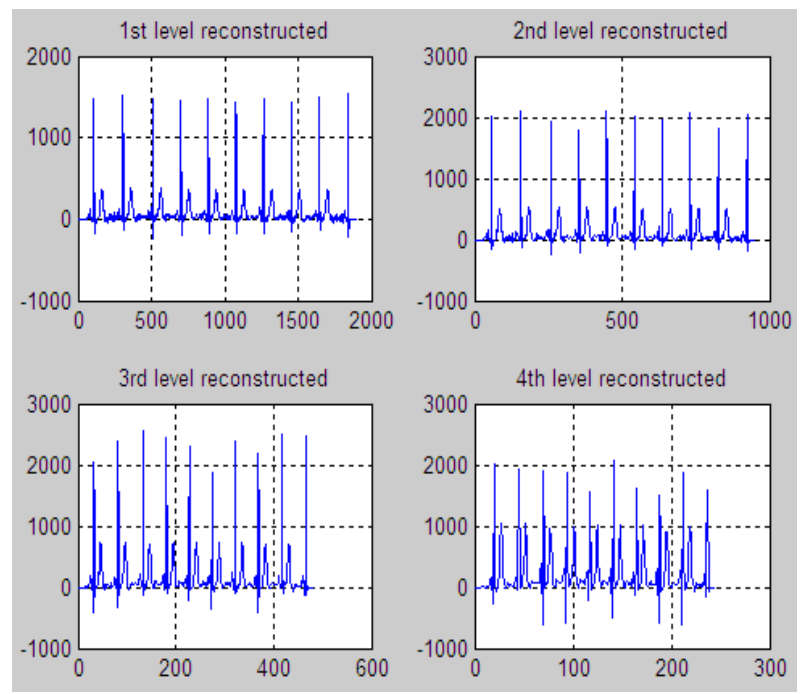

Fig.8 Reconstructed signal

This fig. 8 shows the Reconstructed signal. After proposed algorithm are applied on the input signal and decomposition method are process then we get the reconstructed signal.

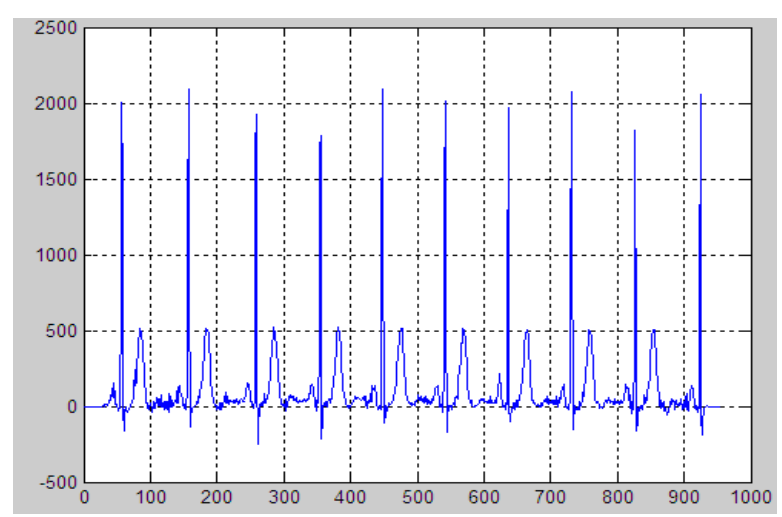

Fig.9 smoothed signal

This fig.9 shows the smoothed signal. 


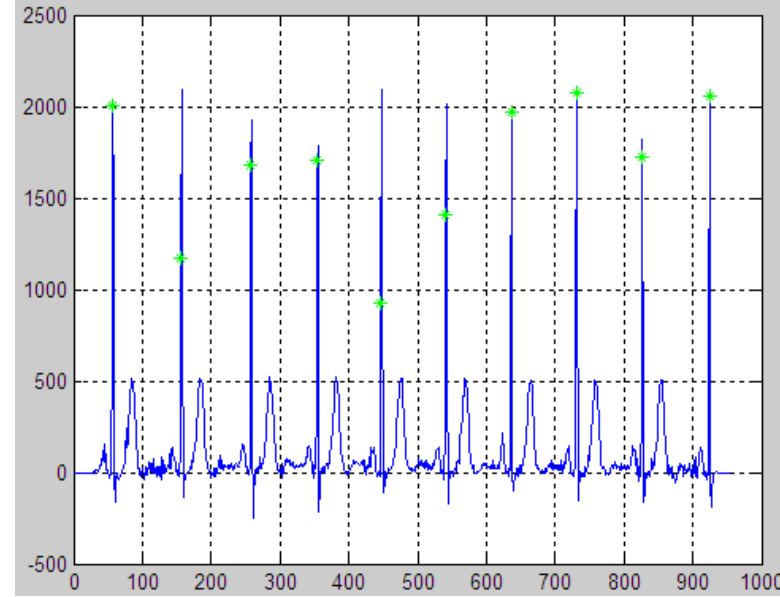

Fig.10 output

This fig. 10 shows the final output signal.

Table 1 Comparison table

\begin{tabular}{|c|c|c|}
\hline $\begin{array}{c}\text { Signal to noise } \\
\text { ratio (SNR) }\end{array}$ & $\begin{array}{c}\text { Previous } \\
\text { Result }\end{array}$ & Proposed Result \\
\hline White SNR & - & 0.2100 \\
\hline Color SNR & 1.0678 & 1.9700 \\
\hline Muscleartifact SNR & 1.1234 & 3.2100 \\
\hline
\end{tabular}

In the above table the SNR values are calculated. Calculate the SNR improvement values and analysis which proposed method gives the high SNR values. In this paper Color SNR and muscle artifact SNR values are compared with the base paper SNR values.

\section{CONCLUSION}

In this paper implemented the energy efficient Power Spectral Analysis of ECG signal using DWT and FFT. Initially, we tend to calculated a domain (i.e. Wavelet) within which the input is about sparse and that we continued by expressing it during this domain using a appropriate transformation (i.e. DWT). The few large signal elements also are most significant/relevant for the general signal quality, whereas different signal elements and also the associated computations will potentially be skipped/pruned for big complexness reduction. FFT compete the most vital role to analyses the spectral coefficients of ECG.

Simultaneously diagnostic \& prognostic importance of wavelet techniques in various field of ECG needs to be recognized in large clinical studies. moreover the work can be further develop by disease diagnostic schemes for ECG \& EEG signals.

\section{REFERENCES}

[1] Vijayakumari, B., J. Ganga Devi, and M. Indhu Mathi. "Analysis of noise removal in ECG signal using symlet wavelet." Computing Technologies and Intelligent Data Engineering (ICCTIDE), International Conference on. IEEE, 2016.

[2] Awal, Md Abdul, et al. "An adaptive level dependent wavelet thresholding for ECG denoising." Biocybernetics and Biomedical Engineering 34.4 (2014): 238-249.
[3] Lin, H-Y., et al. "Discrete-wavelet-transform-based noise removal and feature extraction for ECG signals." IRBM 35.6 (2014): 351-361.

[4] Zivanovic, Miroslav, and Miriam González-Izal. "Simultaneous powerline interference and baseline wander removal from ECG and EMG signals by sinusoidal modeling." Medical engineering \& physics 35.10 (2013): 1431-1441.

[5] Das, M. K., and S. Ari. "Analysis of ECG signal denoising method based on S-transform." Irbm 34.6 (2013): 362-370.

[6] Poungponsri, Suranai, and Xiao-Hua Yu. "An adaptive filtering approach for electrocardiogram (ECG) signal noise reduction using neural networks." Neurocomputing 117 (2013): 206-213.

[7] Chandrakar, Bhumika, O. P. Yadav, and V. K. Chandra. "A survey of noise removal techniques for ECG signals." International Journal of Advanced Research in Computer and Communication Engineering 2.3 (2013): 1354-1357.

[8] Kabir, Md Ashfanoor, and Celia Shahnaz. "Denoising of ECG signals based on noise reduction algorithms in EMD and wavelet domains." Biomedical Signal Processing and Control 7.5 (2012): 481-489.

[9] Martínez, Juan Pablo, et al. "A wavelet-based ECG delineator: evaluation on standard databases." IEEE Transactions on biomedical engineering 51.4 (2004): 570-581.

[10] Ziarani, Alireza K., and Adalbert Konrad. "A nonlinear adaptive method of elimination of power line interference in ECG signals." IEEE transactions on biomedical engineering 49.6 (2002): 540-547.

\section{AUTHOR PROFILE}

Ms. Anamika Rajput has received her Engineering degree in Electronics \& Communication in June 2013 from Rajiv Gandhi Proudyogiki Vishwavidyalaya (RGPV), Bhopal, (M.P.) India and currently pursuing Master of Technology degree in Digital Communication from Technocrats Institute of Technology- Advance under Rajiv Gandhi Proudyogiki Vishwavidyalaya (RGPV), Bhopal (M.P.) India.

Prof. Pankaj Soni has received his Engineering degree in June 2008 and Master of Technology degree in Dec 2012 from Rajiv Gandhi Proudyogiki Vishwavidyalaya (RGPV), Bhopal, (M.P.) India. He is currently working as Professor in department of Electronics \& Communication in Technocrats Institute of Technology- Advance, Bhopal (M.P.) India. He has five years teaching experience. He has published 9 international research papers. His research interest is in Digital Communication, Wireless Communication and VLSI Design.

Prof. Anshul Awasthi has received his Engineering degree in June 2009 and Master of Technology degree in June 2012 from Rajiv Gandhi Proudyogiki Vishwavidyalaya (RGPV), Bhopal (M.P.) India. He is currently working as Professor in department of Electronics \& Communication in Technocrats Institute of Technology- Advance, Bhopal, (M.P.) India. He has five years teaching experience. His research interest is in Digital Communication and VLSI Design. 